Low levels of CT positivity in this cohort demonstrate the importance of further investigations. Correct pNGU treatment may also lead to fewer visits and reduced burden on the service.

\section{P087 WHERE HAS ALL THE MYCOPLASMA GONE?}

${ }^{1}$ Tadiwanashe Chirawu*, 'Rohilla Maarij, ${ }^{2}$ Elizabeth Foley, ${ }^{1,2}$ Raj Patel. 'Southampton Medical School, Southampton, UK; ${ }^{2}$ Royal South Hants Hospital, Southampton, UK

\subsection{6/sextrans-2016-052718.141}

Background Non gonococcal urethritis (NGU) is thought to be most often due to Chlamydia trachomatis (CT), Mycoplasma genitalium (MG) and Ureaplasma urealyticum. Standard doses of Azithromycin $1 \mathrm{~g}$ stat may be insufficient to clear MG and may induce resistance to macrolides while doxycycline is not sufficient to clear MG. Doxycycline has been advocated as first line therapy for NGU allowing extended dosage with Azithromycin in those that do not clear with first line therapies.

Aim To look at the current pathways for managing NGU and assess how often patients who have first line Doxycycline return for further therapy.

Methods A retrospective review of all patients initially treated for NGU in August and September 2015 across a sexual health trust.

Results Of the 208 cases reviewed, 26.4\% were due to CT. $99.5 \%$ of all cases were treated with a first line antibiotic; with 95.2\% receiving Doxycycline $100 \mathrm{mg}$ bd for 7 days compared to $4.3 \%$ receiving Azithromycin $1 \mathrm{~g}$ stat. In both the CT and nonCT groups 9\%, returned within 90 days after experiencing symptoms despite treatment. Of these, only $15.8 \%$ were diagnosed with persistent NGU and treated with extended Azithromycin.

Conclusion Current pathways designed to preserve macrolide therapy using Doxycycline initially result in few patients reattending with persistent symptoms than would be anticipated. The impact of Doxycycline on reducing MG load and related symptoms should be factored into advice given to patients who may dismiss mild symptoms or be reassured by negative CT/NG NAATs often communicated to them by text.

\section{P088 SEXUAL CONTACT IS THE TRIGGER! WOMEN'S VIEWS AND EXPERIENCES OF THE TRIGGERS FOR THE ONSET OF BACTERIAL VAGINOSIS AND EXACERBATING FACTORS ASSOCIATED WITH RECURRENCE}

1,3 Jade Bilardi, 1,35andra Walker, ${ }^{4}$ Julie Mooney-Somers, ${ }^{2}$ Ruth McNair, ${ }^{2}$ Meredith TempleSmith, ${ }^{3,1}$ Clare Bellhouse, ${ }^{1,3}$ Christopher Fairley* ${ }^{1,3}$ Marcus Chen, ${ }^{1,3}$ Catriona Bradshaw. ${ }^{1}$ Central Clinical School, Monash University, Melbourne, Australia; ${ }^{2}$ Department of General Practice, The University of Melbourne, Melbourne, Australia; ${ }^{3}$ Melbourne Sexual Health Centre, Alfred Health, Melbourne, Australia; ${ }^{4}$ Centre for Values, Ethics and the Law in Medicine, The University of Sydney, Sydney, Australia; ${ }^{5}$ Melbourne School of Population and Global Health, The University of Melbourne, Melbourne, Australia

\section{$10.1136 /$ sextrans-2016-052718.142}

Background/introduction Bacterial vaginosis (BV) is the most common vaginal infection affecting women of childbearing age. While the aetiology and transmissibility of BV remain unclear, there is strong evidence to suggest an association between BV and sexual activity.

Aim(s)/objectives This study aimed to explore women's views and experiences of the triggers for BV onset and factors associated with recurrence
Methods A descriptive, social constructionist approach was chosen as the framework for the study. Thirty five women of varying sexual orientation who had experienced recurrent BV in the past five years took part in semi-structured interviews.

Results The majority of women predominantly reported sexual contact triggered the onset of BV and sexual and non-sexual factors precipitated recurrence. Recurrence was most commonly referred to in terms of a 'flare-up' of symptoms. The majority of women did not think BV was an STI however many reported being informed this by their clinician. Single women who attributed BV onset to sex with casual partners were most likely to display self-blame tendencies and to consider changing their future sexual behaviour. Women who have sex with women (WSW) were more inclined to believe their partner was responsible for the transmission of or reinfection with BV and seek partner treatment or change their sexual practices.

Discussion/conclusion Findings from this study strongly suggest women believe that BV onset is associated with sexual activity, concurring with epidemiological data which increasingly suggest sexual contact is associated with the development of BV. There was some evidence to suggest possible transmission among WSW reinforcing the need for new approaches to treatment and management strategies.

\section{P089 DETECTION OF NEISSERIA GONORRHOEAE BACTERIAL LOADS IN THE PHARYNX AND SALIVA AMONG MEN WHO HAVE SEX WITH MEN}

\begin{abstract}
${ }^{1,2}$ Eric Chow* ${ }^{3,4}$ Sepehr Tabrizi, ${ }^{3,5}$ Samuel Phillips, ${ }^{1}$ David Lee, ${ }^{1,2}$ Catriona Bradshaw,
${ }^{1,2}$ Marcus Chen, ${ }^{1,2}$ Christopher Fairley. 'Melbourne Sexual Health Centre, Alfred Health, Melbourne, VIC, Australia; ${ }^{2}$ Central Clinical School, Faculty of Medicine, Nursing and Health Sciences, Monash University, Melbourne, VIC, Australia; ${ }^{3}$ Microbiological Diagnostic Unit Public Health Laboratory, Department of Microbiology and Immunology, The University of Melbourne, at the Peter Doherty Institute for Infection and Immunity, Melbourne, VIC, Australia; ${ }^{4}$ Department of Microbiology and Infectious Diseases, The Royal Women's Hospital, Parkville, VIC, Australia; ${ }^{5}$ Department of Obstetrics and Gynaecology, University of Melbourne, Parkville, VIC, Australia; ${ }^{6}$ Murdoch Childrens Research Institute, Parkville, VIC, Australia
\end{abstract}

\subsection{6/sextrans-2016-052718.143}

Background/introduction Studies have shown that N. gonorrhoeae can be cultured in human saliva among individuals with pharyngeal gonorrhoea. As saliva could potentially act as a carrier for gonorrhoea transmission during sex, the bacterial load of $N$. gonorrhoeae in saliva may influence the transmissibility of gonorrhoea.

Aim(s)/objectives To quantify the gonococcal bacterial load in the pharynx and saliva among men who have sex with men (MSM) with untreated pharyngeal gonorrhoea.

Methods MSM who tested positive for pharyngeal gonorrhoea by culture were recalled for antibiotic treatment within 14 days between October 2014 and March 2015. The gonococcal bacterial load was estimated using real-time quantitative PCR (qPCR) by interpolating against a standard curve generated with known gonococcal DNA copy numbers. The median of gonococcal bacterial load in the pharynx and saliva was calculated and compared between culture positivity using Mann-Whitney $U$ test.

Results A total of 33 men were included in this study. At the time of treatment, the median gonococcal bacterial load in the pharynx was similar in men who were culture-positive $(2.5 \times$ $10^{5}$ copies/swab) and culture-negative $\left(2.9 \times 10^{4}\right.$ copies/swab) $(p=0.166)$, and similar in the saliva in culture-positive: $2.2 \times$ 